\title{
BMJ Open LASI-DAD study: a protocol for a prospective cohort study of late-life cognition and dementia in India
}

\author{
Jinkook Lee, ${ }^{1}$ Joyita Banerjee, ${ }^{2}$ Pranali Yogiraj Khobragade, ${ }^{1}$ Marco Angrisani, ${ }^{1}$ \\ A B Dey ${ }^{2}$
}

To cite: Lee J, Banerjee J, Khobragade PY, et al. LASIDAD study: a protocol for a prospective cohort study of late-life cognition and dementia in India. BMJ Open 2019;9:e030300. doi:10.1136/ bmjopen-2019-030300

- Prepublication history for this paper is available online. To view these files, please visit the journal online (http://dx.doi. org/10.1136/bmjopen-2019030300).

Received 8 March 2019 Revised 27 June 2019 Accepted 3 July 2019

Check for updates

(c) Author(s) (or their employer(s)) 2019. Re-use permitted under CC BY-NC. No commercial re-use. See rights and permissions. Published by BMJ.

${ }^{1}$ Dana and David Dornsife College of Letters, Arts and

Sciences, University of Southern California, Los Angeles, California, USA

${ }^{2}$ Geriatric Medicine, All India Institute of Medical Sciences, New Delhi, Delhi, India

Correspondence to Dr. Jinkook Lee; jinkookl@usc.edu and Professor A B Dey; abdey@hotmail.com

\section{ABSTRACT}

Introduction Alzheimer's disease and related dementias can be considered the epidemic of the 21st century. Particularly, the predicted growth in the size of elderly populations in low-income and middle-income countries is expected to produce a dramatic surge in dementia prevalence and incidence. Although a rising burden of dementia presents an urgent challenge for India, previous efforts to study dementia in the country have relied on non-representative samples in geographically restricted regions. The Harmonised Diagnostic Assessment of Dementia for the Longitudinal Aging Study in India (LASIDAD) will provide rich, population-representative data on late-life cognition and dementia and their risk factors for the first time in India.

Methods The LASI-DAD will recruit a sample of 3000 people aged $60+$ years. Their family members or friends, whom respondents nominate as informants, participate in the computer-assisted personal interview. The study sample is drawn from the ongoing, nationally representative Longitudinal Aging Study in India, a multipurpose panel survey of aging. We aim to collect rich data on cognitive and neuropsychological tests, informant reports, and epidemiological data through a comprehensive geriatric assessment, and venous blood collection and assays. For a subsample, we collect neuroimaging data. Data collection is currently in progress in 14 States and Union Territories of India. Clinicians will provide clinical consensus diagnosis based on the Clinical Dementia Rating.

Ethics and dissemination Ethics approval was obtained from the Indian Council of Medical Research and all collaborating institutions. Anonymised data will be available for the larger research community through a secured website hosted by the Gateway to Global Aging Data platform. Research findings from the LASI-DAD team will be disseminated through journal publications and presentations at professional conferences.

\section{INTRODUCTION}

Alzheimer's disease and related dementias (hereafter dementia) can be considered the epidemic of the 21st century. In 2017, there were an estimated 50 million people worldwide living with dementia. In 2010, the Alzheimer's and Related Disorders Society of India $(\text { ARDSI })^{1}$ estimated that more than

\section{Strengths and limitations of this study}

- The Harmonised Diagnostic Assessment of Dementia for the Longitudinal Aging Study in India (LASI-DAD) will provide population-representative data on cognition and dementia for the first time in India.

- LASI-DAD uses the Harmonized Cognitive Assessment Protocol, a common set of cognitive and neuropsychological test batteries used by an international network of researchers, enabling cross-country comparisons.

- LASI-DAD collects rich epidemiological data through a comprehensive geriatric assessment, venous blood collection and assays; for a subsample of 200 respondents, neuroimaging data collection is in progress.

- Pathologically based, inperson diagnosis of dementia is not available, but clinicians will provide the Clinical Dementia Rating based on the LASI-DAD data.

- Data will be collected from 14 States and Union Territories of India, representing $72 \%$ of the population. We will construct sample weights to represent the population.

3.7 million Indians had dementia, a number comprising more than $10 \%$ of all persons worldwide with dementia. This result is, however, based on meta-analysis of all population-based prevalence studies in the prior decade. These studies reported prevalence rates ranging from $0.6 \%$ to $10.6 \%$ in rural areas and from $0.9 \%$ to $7.5 \%$ in urban areas. The heterogeneity in reported prevalence could be due to a variety of methodological issues, including regional differences and different diagnostic criteria.

Although a rising burden of dementia presents an urgent challenge for India, previous efforts to study dementia in the country have relied on non-representative samples in geographically restricted regions. For example, landmark studies by Ganguli $e a^{2}{ }^{23}$ interviewed 374 individuals aged 55 and older at a rural site near Delhi in the state of 
Haryana, and the 10/66 Dementia Research Group's estimate of dementia prevalence was based on a multicentre convenience sample of 760 respondents aged 60 and older from six states. ${ }^{4}$ The Harmonised Diagnostic Assessment of Dementia for the Longitudinal Aging Study in India (LASI-DAD) overcomes this limitation by interviewing a population-representative sample, using the sampling framework of the Longitudinal Aging Study in India (LASI), an ongoing prospective panel study, representative of the nation as well as each state.

LASI is the most ambitious, nationally representative survey of the physical and cognitive health, economic, and social well-being for the country's aging population, interviewing over 70000 individuals aged 45+ (including their spouses, irrespective of age). The National Institute on Aging (R01AG042778) and the Government of India (Ministry of Health and Family Welfare) have provided funding for two waves of data collection. No other large, longitudinal survey in India collects cognition data as rich and detailed as LASI. LASI-DAD extends LASI's cognition data collection by administering indepth cognitive tests and informant interviews to a subsample of 3000 LASI respondents aged 60 or older, closely following the Harmonized Cognitive Assessment Protocol (HCAP).

The HCAP was developed for the assessment of dementia and mild cognitive impairment (MCI) in the US Health and Retirement Study and its sister studies around the world ${ }^{5}$ to enable international research collaboration. It is based on best-practice instruments for multiple cognitive domains and informant reports and designed for flexible statistical harmonisation. It has been used successfully in the USA, England, Mexico, China and South Africa. Through multiple pretests, we have carefully evaluated the HCAP in a local Indian setting, making culturally appropriate modifications and adding cognitive tests that will enable comparison with prior studies in India.

The impact of dementia on individuals, families and society is expected to increase rapidly with population ageing in India. The ARDSI ${ }^{1}$ estimates the total societal cost of dementia in India at US\$3.4 billion (147 billion Indian rupees). This estimate is based on Wimo and Prince's ${ }^{6}$ approach to calculating the cost of dementia as time taken by caregivers multiplied by the average national wage. The need for more accurate estimates of the burden of the disease using high-quality economic data is paramount. ${ }^{17}$ LASI-DAD is ideal for such estimates, as rich data on economic resources, healthcare access and use, labour force behaviours, and caregiving arrangements are available from LASI-DAD combined with the main LASI.

India provides an excellent epidemiological 'laboratory' to study key risk factors for dementia and MCI. The high prevalence of cardiovascular risk factors (eg, hypertension, diabetes and hyperlipidaemia) and extremely high levels of ambient air pollution in certain cities in India provide an unparalleled opportunity to also study these important risk factors. LASI-DAD collects rich epidemiological data through geriatric assessments and venous blood specimen (VBS) collection. For a subsample $(n=200)$, LASI-DAD collects structural and resting-state functional MRI data, following the Alzheimer's Disease Neuroimaging Initiative (ADNI) 3; for a larger subsample $(n=960)$, we conduct genotyping, using the Illumina Infinium Global Screening Array-24 V.2.0 (GSA) BeadChip. The main LASI also collects rich data on individual characteristics, including health, health behaviours, access to and use of healthcare, exposure to indoor air pollution, and a wide range of demographic information as well as information on respondents' financial situation and residential history. We will also collect latitude and longitude for all households we interview, which we will use to calculate respondents' exposure to ambient air pollution.

\section{METHODS AND ANALYSIS \\ Study aims}

An overarching goal of LASI-DAD is to advance dementia research by collecting and analysing high-quality data on late-life cognition, dementia and MCI and their correlates. Our project team aims the following:

1. Collect high-quality data on late-life cognition and dementia $(n=3000)$.

2. Enrich epidemiological data through geriatric assessment, VBS collection and assays, neuroimaging, and genotyping.

3. Obtain clinical consensus diagnosis.

4. Estimate the prevalence of dementia and MCI.

5. Investigate the determinants of late-life cognition, dementia and MCI.

6. Study the impact of dementia and MCI on families and society.

7. Disseminate anonymised data to larger research community.

\section{Study design}

Our study protocol consists of cognitive tests, informant interview, geriatric assessment, VBS-based assays, neuroimaging and genotyping.

After careful evaluation and multiple pretests, we have included in our cognitive test protocol a set of cognitive and neuropsychological test batteries common with the Health and Retirement Study (HRS)-Harmonized Cognitive Assessment Protocol (HCAP)..$^{5}$ These include object naming, ${ }^{8}$ the Consortium to Establish a Registry for Alzheimer's Disease word recall, ${ }^{9}$ backward digit span, ${ }^{10}$ logical memory, ${ }^{11}$ constructional praxis, ${ }^{12}$ retrieval fluency, ${ }^{9}$ serial $7 \mathrm{~s},{ }^{13}$ Community Screening Instrument for Dementia (CSI-D) ${ }^{14}$ and Raven's test. ${ }^{15}$ Several of these tests were also included in the main LASI, allowing calibration. Table 1 presents the test batteries we employed in comparison with the main LASI cognition module and the domains measured. We dropped backward counts ${ }^{16}$ and number series, ${ }^{17}$ which were administered in the main LASI, as these tests were proven to be very difficult 
Table 1 LASI-DAD cognitive test protocols in comparison with LASI cognitive test protocol

\begin{tabular}{|c|c|c|}
\hline Test selected in LASI & Domains measured & Administered in DAD \\
\hline $\begin{array}{l}\text { Word recall (one trial): immediate and } \\
\text { delayed }^{9}\end{array}$ & Memory & $\begin{array}{l}\text { Three-trial word learning: immediate, } \\
\text { delayed, recognition }\end{array}$ \\
\hline $\begin{array}{l}\text { TICS: date, place, naming objects, prime } \\
\text { minister }\end{array}$ & Orientation, language & $\begin{array}{l}\text { Yes, date and place orientation is a part } \\
\text { of HMSE; and object naming }\end{array}$ \\
\hline MoCA: clock drawing only ${ }^{35}$ & Executive function, visuospatial skills & Yes \\
\hline Backward counting $^{16}$ & Numeric ability, attention & Dropped after phase 1 \\
\hline Number series ${ }^{17}$ & Numeric ability, fluid intelligence & No \\
\hline
\end{tabular}

LASI, Longitudinal Aging Study in India; LASI-DAD, Harmonised Diagnostic Assessment of Dementia for the Longitudinal Aging Study in India; HMSE, Hindi Mental State Examination; MMSE, Mini Mental State Examination; MoCA, Montreal Cognitive Assessment; TICS, Telephone Interview for Cognitive Status.

to administer, as the large population was innumerate. We also included additional tests designed for illiterate and innumerate populations, including the Hindi Mental State Exam, ${ }^{2}$ symbol cancellation ${ }^{18}$ and Go-No Go test. ${ }^{19}$ Table 2 presents additional cognitive tests included in LASI-DAD.

Informant interview is another key component of the HCAP. As the HRS-HCAP does, LASI-DAD asks respondents to nominate a close family member or friend as an informant who knows the respondent well, interacts with the respondent frequently, and therefore knows the respondent's daily functions and can report on them. Informants are most likely to be spouses, partners, children or a long-time caregiver. The informant interview consists of questions about the respondent's functional status, social engagements and memory, including the Informant Questionnaire on Cognitive Decline in the Elderly (IQCODE), ${ }^{20}$ the Blessed Dementia Rating Scale, $^{21}$ the CSI-D Cognitive Activities Questionnaire ${ }^{14}$ and the 10/66 Dementia Research Group Informant Questionnaire. $^{22}$

The LASI-DAD geriatric assessment includes specific physical measures such as blood pressure, anthropometry, questions on functional limitations, anxiety ${ }^{23}$ and depression, ${ }^{24}$ and tests of functionality, including Timed Up and Go Test, ${ }^{25}$ Six-Minute Walk Test, ${ }^{26}$ nutrition ${ }^{27}$ and hearing using a HearCheck device.

LASI-DAD also collects and assays VBS in partnership with Metropolis Laboratory, a leading independent pathology laboratory in India accredited by the National Accreditation Board for Testing and Calibration Laboratories. Specifically, LASI-DAD collects a $17 \mathrm{~mL}$ fasting blood from each participant. The blood-based assays being done are complete blood counts, glycosylated haemoglobin, lipid panel, lipoprotein A, metabolic panel, homocysteine, vitamin $\mathrm{B}_{12}$, folic acid, vitamin $\mathrm{D}$, thyroid stimulating hormone, high-sensitivity $\mathrm{C}$ reactive protein and N-terminal pro-B-type natriuretic peptide.

Table 2 Additional tests administered in LASI-DAD

\section{Additional tests selected in LASI-DAD}

Hindi Mental State Exam ${ }^{2}$

Digit span forward ${ }^{10}$

Symbol cancellation $^{18}$

Logical memory: immediate, delayed, recognition ${ }^{11}$

Constructional praxis: copy, delayed recall ${ }^{12}$

Hand sequence ${ }^{36}$

Token test $^{37}$

Judgement, problem solving ${ }^{30}$

Community Screening Instrument for Dementia ${ }^{14}$

Raven's test ${ }^{15}$

Go-No Go test ${ }^{19}$

\section{Domains measured}

Orientation, memory, visuospatial, attention/speed, language/fluency Attention/speed

Attention/speed

Memory

Visuospatial skills, memory

Executive functioning

Language/fluency

Judgement, executive functioning

Orientation, executive functioning, language/fluency

Executive functioning

Executive functioning

LASI-DAD, Harmonised Diagnostic Assessment of Dementia for the Longitudinal Aging Study in India. 
For a subsample of 200, the LASI-DAD protocol collects neuroimaging data on a pilot basis. This is the first attempt to collect neuroimaging data for a population survey. In collaboration with the University of Southern California Laboratory of Neuro Imaging team, the LASI-DAD team is employing the ADNI 3 protocol (see http://adni.loni. usc.edu/adni-3/ for details). The scan protocol, which consists of structural brain imaging and resting-state functional MRI, requires around $55 \mathrm{~min}$ to complete.

\section{Patient and public involvement}

Patients and the public are not involved in the design and recruitment of this project.

\section{Sample}

The LASI-DAD sample was drawn from the LASI main study sample, which is representative of both the nation at large and each state. Our target sample is 3000 persons aged 60 years and above.

To obtain national representation within the budgetary constraints, as well as quality supervision of the fieldwork, we have recruited 11 centres which include 9 regional geriatric centres (RGCs), 1 apex neuroscience centre and 1 partner centre to collaborate in interviewer recruitment and fieldwork management. These are All India Institute of Medical Sciences, Bhubaneshwar and New Delhi; Dr SN Medical College, Jodhpur; Government Medical College, Thiruvananthapuram; Grant Medical College and JJ Hospital, Mumbai; Guwahati Medical College, Guwahati; Institute of Medical Sciences, Banaras Hindu University, Varanasi; Madras Medical College, Chennai; Medical College, Kolkata; National Institute of Mental Health and Neurosciences (NIMHANS), Bengaluru; Nizam's Institute of Medical Sciences, Hyderabad; and Sher-e-Kashmir Institute of Medical Sciences, Srinagar. In consideration of proximity to these RGCs, we selected the sample from 14 states across the country, representing $72.2 \%$ of the population. These 14 states include Assam, Delhi, Haryana, Jammu and Kashmir, Karnataka, Kerala, Madhya Pradesh, Maharashtra, Odisha, Rajasthan, Tamil Nadu, Telangana, Uttar Pradesh and West Bengal.

A simple random sample of age-eligible LASI respondents would not yield a sufficient number of cognitively impaired respondents for a reasonably precise estimation of the relationship between dementia and its correlates. Therefore, we employ a two-stage stratified sampling approach. First, we assign sample quota to each of the 14 participating states proportional on their population. Second, we classify respondents into high and low risk of cognitive impairment using the LASI main study's cognitive tests and proxy report for those who did not complete the cognitive tests. Specifically, we determine whether respondents are at high risk of cognitive impairment if any of the following conditions are met: (1) overall cognitive test performance in the main LASI is in the bottom tertile; (2) memory score is below the 15 th percentile; (3) non-memory cognitive scores are below the 15 th percentile; (4) number of missing cognitive tests is above the 85th percentile; or (5) the IQCODE score ${ }^{20}$ is 3.9 or higher. Once individuals are classified as having either low or high risk of cognitive impairment, we randomly draw an equal number of individuals from these two mutually exclusive groups within the predefined sample quota for each state. Our sampling strategy leads to an oversample of individuals with high risk of cognitive impairment, which should ensure sufficient power to estimate dementia and MCI prevalence and to detect statistical associations between these conditions and potential risk factors.

We create sample weights that account for differential selection probabilities produced by the adopted sampling strategy and adjust for differential non-response. In a first step, we compute a design weight that accounts for the oversampling of individuals classified as having high risk of cognitive impairment. Starting from these design weights, we then apply a raking algorithm to produce poststratification weights. The resulting sample weights align sample distributions of key demographic variables to their population benchmarks. Specifically, the following variables are used as raking factors: gender (male/female) $\times$ age $(60-69 / 70+)$; gender $\times$ literacy (literate/illiterate); and urbanicity (rural/urban). Benchmark distributions for these variables are taken from the Indian Census 2011 and refer to the population of individuals aged 60 and above in all 14 LASI-DAD states.

Sample weights allow to make inference about the reference population-individuals aged 60 and above in the 14 LASI-DAD states-which constitutes $72.2 \%$ of the entire Indian population aged 60 and above. The generalisability of the LASI-DAD results to the entire Indian population aged 60 and above is subject to some caveats. While demographic differences between LASI-DAD included and excluded states can be corrected for by poststratifying to the entire Indian population aged 60 and above, the population of older adults in the included LASI-DAD states may differ from that of the excluded LASI-DAD states in terms of MCI and dementia risk. In the absence of population benchmarks for MCI and dementia risk, these differences cannot be accounted for. However, data from the main LASI can, at least partially, document such differences and inform about the extent to which inference from the LASI-DAD study to the entire Indian population aged 60 and above is appropriate.

\section{Recruitment and fieldwork}

At least 2 months after the LASI baseline interview, we recruit the selected respondents and their informants for an interview. As the LASI baseline interviews were fielded in three phases, we planned DAD fieldwork about 6 months afterwards. Phase 1 fieldwork started in October 2017 and ended in June 2018. We are currently in phase 2 fieldwork, which started in October 2018. Our field team consists of four to five clinical psychologists, nurses and medical social workers who personally visit the selected respondents. Based on respondents' preference and their proximity to the hospital, the interview team administers the HCAP protocol either at the hospital or at the 
respondent's home. The field team sometimes travel up to 12 hours by automobile to reach respondents residing in remote villages.

We pay inperson visit to selected LASI-DAD respondents and ask for their participation. Selected respondents may or may not participate. All consent procedures were approved by the study's ethics committees. For selected respondents who refuse to participate, we ask them to nominate informants and conduct informant interview. To encourage respondents' participation, we offer token cash remuneration. We also arrange for transportation of participants to and from the hospital for hospital interviews and neuroimaging study. We provide meals and refreshments to respondents after blood draws and lab results of blood tests when complete.

Fieldwork comprises a face-to-face computer-assisted personal interview (CAPI), geriatric assessment and venous blood draw. The face-to-face CAPI interview takes about 1 hour for cognitive tests and another 35-40 min for geriatric assessment. The $17 \mathrm{~mL}$ VBS is collected by a phlebotomist where the cognitive interview occurs, either at the respondent's home or at participating hospitals. When the VBS is drawn at home, a separate visit by a phlebotomist is arranged to obtain fasting blood. Once preliminary processing of the blood is done at local laboratory, cold-chained blood is transported to the central laboratory in Delhi. A small amount of blood $(2.5 \mathrm{~mL})$ is transported to an earmarked genetic laboratory, MedGenome, for genomic work. We genotyped the phase 1 sample, using Global Screening Array, and are currently seeking funding for whole genome sequencing of the entire study sample. The central Metropolis Laboratory segregates the samples after receiving them for analysis, and sends serum, plasma, buffy coat and dried blood spot cards to the Department of Geriatric Medicine, All India Institute of Medical Sciences (AIIMS), New Delhi for storage in anticipation of future studies.

A separate visit is arranged for a neuroimaging study based on geographical proximity to neuroimaging facilities. For this neuroimaging study we have recruited two partner institutions based on equipment availability (3.0 Tesla with 32 head-coils) and commitment to achieving high-quality imaging data at low cost. These institutions are the NIMHANS in Bengaluru and the NM Medical Centre in Mumbai. We give additional cash remuneration to participants in the neuroimaging study and arrange round-trip transportation to facilitate their participation. All respondents who complete the cognitive tests and reside within 4-hour distance by automobile to the partner neuroimaging site are asked to participate in the neuroimaging study.

All the field teams administering the HCAP receive 2-week training. The training workshop includes the following content: project overview, locating, approaching and obtaining consent from the study subjects, how to conduct CAPI, interviewing rules and techniques required for administering neuropsychological tests, geriatric assessments, and informant interviews, paying incentive to the study subjects and keeping receipts, and data uploads.

During the fieldwork, the field management team is in close contact with the coordinators at each site, monitoring the progress and solving any potential issues the interviewers encounter. As the CAPI data are uploaded into a central server, our data management monitors data on a weekly basis, downloading the data and examining the distribution. Specifically, we pay attention to missing data and patterns of data distribution across sites, interviewers, and respondent characteristics such as age and literacy.

\section{Consent}

Consent to participate comprises the following: (1) respondents' consent to participate in the cognitive test, geriatric assessment, VBS draw, assays and genomic work; (2) respondents' consent to participate in neuroimaging study; and (3) informants' consent to participate in informant interview. Consent is directly taken from the respondents. For respondents who are cognitively impaired, we will obtain consent from a close family member, such as a spouse or adult child who can legally represent the respondent. If the respondent is unable to read the consent forms, the interviewer will read them to the respondent. Respondents who are unable to sign the consent forms may use a thumb impression in place of a signature. The consent materials will be translated into as many local languages as necessary. Consent and interviews will be collected and conducted in the respondent's language.

\section{Clinical consensus diagnosis}

For conditions such as Alzheimer's disease, dementia and MCI, there is no single definitive diagnostic test. To identify such conditions in respondents, many clinical researchers rely on a process of data review, adjudication and consensus by a panel of expert clinicians. ${ }^{28}$ The panel meets in person to review detailed information on various aspects of the clinical assessment of a given patient, discusses the findings, and renders a consensus diagnosis using standardised criteria. This process allows the data of each study participant to be individually considered in detail, taking advantage of a wealth of collective clinical expertise and judgement. However, it involves the cost of the time spent by experts in examining the patients, the inefficiency of scheduling meetings at a time and location that all experts can attend, and the near impossibility of including experts at different sites. For large population surveys, the standard of 'clinician's in-person assessment' of the respondents and all relevant information from their informants and 'in-person consensus conference' is too costly to implement.

Building on the online consensus approach developed by the Monongahela-Youghiogheny Healthy Aging Team, ${ }^{28}{ }^{29}$ we built an online consensus website. We present the information and data from the LASI-DAD study organised according to the Clinical Dementia 
Rating (CDR) subdomains and present them to clinicians for CDR rating. ${ }^{30}$ For each case, three to four clinicians are assigned for the first individual review. Each clinician reviews each assigned case and provides domain-specific ratings. Following the CDR algorithm, global rating is generated for each individual reviewer, and all assigned reviewers' global ratings are then compared. For cases where individual global ratings differ, an automatic email goes out to assigned reviewers, giving a chance to review the case and read other raters' comments and update their ratings, if desired. After this second review, consensus may be reached. For cases where consensus was not reached, a group of clinicians discuss the case through a virtual consensus meeting.

We conducted a feasibility of online consensus study in 2018, with the goal of obtaining clinicians' evaluation of whether the clinical consensus website we developed provides adequate information for dementia and MCI diagnosis. For the feasibility study, we identified five patients the clinicians at the NIMHANS have recently diagnosed, including two with dementia, two with MCI and one cognitively normal. Following the LASI-DAD protocol, our team interviewed these five patients and their family members, uploading the collected data on the secured server, which were then fed into the online consensus website. We then invited eight clinicians for online consensus review and asked them to evaluate each case and provide the CDR rating. All participating clinicians, who are certified after receiving formal CDR training, concluded that online consensus diagnosis is a viable alternative and provided further advice to improve the site.

Having made the recommended changes, we are currently conducting a validation study to compare the agreement in clinical consensus diagnosis reached in person and online. We will calculate the kappa interrater agreement measure between the consensus diagnoses reached online and in person. A kappa value of 0.75 is generally considered excellent, and values between 0.40 and 0.75 are considered fair to good agreement. ${ }^{31}$ According to a power analysis using the fractions above and a standard underlying psychometric model, we find that a sample size of 60 cases allows for a nearly $90 \%$ chance of obtaining a kappa of 0.75 or higher if the population kappa of the process is 0.80 .

Once the validity of the online consensus panel is demonstrated, we aim to carry out online clinical reviews for the entire LASI-DAD sample. These data will constitute an important source for the study of late-life cognition and dementia; and their risk factors. Such a purpose requires a sufficient sample to be clinically examined, so that when the data are released other researchers can use them to test their own hypotheses, which may be more complex than what we may envision. If the validation study suggests significant differences between the online consensus diagnosis and inperson clinical assessment, we will obtain inperson clinical assessments for the maximum sample size that we can achieve within our budget.

\section{Data analysis}

We aim to estimate the prevalence of dementia and MCI. Depending on the results of the validation study, we will obtain either inperson or online clinical consensus diagnosis for a subset of or the entire LASI-DAD sample. Therefore, the estimation strategy will be contingent on the results of the validation study. Should online clinical consensus diagnosis be validated, we will obtain it for the entire LASI-DAD sample, from which we can then obtain dementia and MCI prevalence rates by applying the poststratified sampling weights based on the 2011 Census.

As discussed above, should the online consensus method not be deemed sufficiently accurate, we will proceed with inperson clinical diagnosis for a subset of LASI-DAD participants and probabilistically predict clinical diagnosis. We will base our model specification on the model used by Hurd et $a l,{ }^{32}$ with some additions because of the richer LASI-DAD data and progressed theoretical insights. The estimation sample for our model is the LASI-DAD sample for which we have clinical diagnosis. The dependent variable of our model will be the five-category CDR classification (normal, MCI and three levels of dementia severity). Because our dependent variable is an ordinal variable, our model will be an ordered probit model. The regressors in our model are variables measured in the survey: the cognitive test scores and proxy assessments, age, sex, education, and various physical health and disability measures. We will investigate whether interaction terms between various predictors and age group or sex substantially increase predictive power, and consider including the main LASI cognition data to increase predictive power.

For the analysis of the risks for and impacts of latelife cognition, dementia and MCI, we will merge the LASI-DAD data with the main LASI data, as the main LASI survey collects detailed information on a wide range of variables that may be directly or indirectly related with cognition and dementia. These include demographics, age, gender, education, caste, health behaviour, participation in social activities and exposures to indoor air pollution. We will estimate the relationship between cognitive health and these factors and how they influence cognition over the life-cycle. We will control for other potentially confounding factors in estimation, such as noise or other interruption during the administration of cognitive test and language of the test administered. We will construct and introduce into the model measures of exposure to ambient air pollution to investigate how pollution may affect cognitive health. Finally, we will study the impact of dementia on families and society by estimating the effects on caregiving and health and long-term care expenditures.

\section{ETHICS AND DISSEMINATION}

LASI-DAD will provide a rich, anonymised data resource to the larger research community through a secured web platform maintained by the Gateway to Global Aging Data 
(g2aging.org/dad). Further dissemination efforts will be made through webinars and exhibits at professional conferences. The LASI-DAD website will be cross-referenced with other HCAP studies. Research findings will be presented at professional conferences and submitted to peer-reviewed journals.

Acknowledgements The authors would like to acknowledge support from David Bloom, P Arokiasamy and the LASI investigative team; Bas Weerman, Sandy Chien, Eileen Crimmins, Arthur Toga, Arie Kapteyn, Urvashi Jain, Brenton Keller and the Gateway to Global Aging Data team; Sharmistha Dey, Alka Mohan Chutani, David Weir (chair of the LASI-DAD Advisory Board), Kenneth Langa, Sara Adar, Sharon Kardia, Jennifer Smith and the HRS-HCAP team; and Perry Hu, Mary Ganguli, Judith Saxton, Nicoletta Caputi, Mathew Varghese, John John, Himanshu Joshi, RR Mohanty, Arvind Jain, Ravikumar Kurup, R Aruna, Lalit Sankhe, Debabrata Goswami, IS Gambhir, Sankha Subhro Chakrabarty, GS Shanthi, Arunanshu Talukdar, Gargi Dasgupta, YS Raju, M Padmaja, Parvaiz Koul, Shailendra Bhadauria, Priya Moorjani, Andrea Ganna, Claire McVoy, Jorge Jovicich, Geeta Chopra and VL Ramprasad.

Contributors $\mathrm{JL}$ and $\mathrm{ABD}$ developed the overall research design protocol and gained funding. JL prepared the first draft. PYK and JB have been involved in all aspects of the study. MA developed a strategy for constructing sample weights. All authors substantially contributed and commented on the manuscript and approved the final version.

Funding This project is funded by the National Institute on Aging and the National Institutes of Health (R01 AG051125, 1RF1AG055273).

Competing interests None declared.

Patient consent for publication Not required.

Ethics approval We obtained ethics approval from the Indian Council of Medical Research and all collaborating institutions, including the University of Southern California; the All India Institute of Medical Sciences, New Delhi; the International Institute for Population Sciences, Mumbai; All India Institute of Medical Sciences, Bhubaneswar; Dr SN Medical College, Jodhpur; Government Medical College, Thiruvananthapuram; Grant Medical College and JJ Hospital, Mumbai; Guwahati Medical College, Guwahati; Institute of Medical Sciences, BHU, Varanasi; Madras Medical College, Chennai; Medical College, Kolkata; National Institute of Mental Health and Neurosciences, Bengaluru; Nizam's Institute of Medical Sciences, Hyderabad; and Sher-e-Kashmir Institute of Medical Sciences, Srinagar.

Provenance and peer review Not commissioned; externally peer reviewed.

Open access This is an open access article distributed in accordance with the Creative Commons Attribution Non Commercial (CC BY-NC 4.0) license, which permits others to distribute, remix, adapt, build upon this work non-commercially, and license their derivative works on different terms, provided the original work is properly cited, appropriate credit is given, any changes made indicated, and the use is non-commercial. See: http://creativecommons.org/licenses/by-nc/4.0/.

\section{REFERENCES}

1. Alzheimer's \& Related Disorders Society of India. The Dementia India Report 2010: prevalence, impact, costs and services for dementia: executive summary. ARDSI 2010 ardsi.org/downloads/main\% 20report.pdf.

2. Ganguli M, Ratcliff G, Chandra V, et al. A hindi version of the MMSE: The development of a cognitive screening instrument for a largely illiterate rural elderly population in india. Int $J$ Geriatr Psychiatry 1995;10:367-77.

3. Ganguli M, Chandra V, Gilby JE, et al. Cognitive test performance in a community-based nondemented elderly sample in rural India: the Indo-U.S. Cross-National Dementia Epidemiology Study. Int Psychogeriatr 1996;8:507-24.

4. Prince $M$, Acosta $D$, Chiu $H$, et al. Dementia diagnosis in developing countries: a cross-cultural validation study. Lancet 2003;361:909-17.

5. Weir D, Langa K, Ryan L. Harmonized cognitive assessment protocol (hcap): Study protocol summary. Institute for Social Research University of Michigan 2016 https://hrs.isr.umich.edu/publications/ biblio/9950.

6. Wimo A, Prince M. Alzheimer's disease international, world alzheimer report 2010: The global economic impact of dementia. Alzheimer's Disease International;2010 https://www.alz.co.uk/research/files/Worl dAlzheimerReport2010.pdf.
7. Rao GN, Bharath S. Cost of dementia care in India: delusion or reality? Indian J Public Health 2013;57:71-7.

8. Brandt J, Spencer M, Folstein M. The telephone interview for cognitive status. Cogn Behav Neurol 1988;1:111-8.

9. Morris JC, Heyman A, Mohs RC, et al. The Consortium to Establish a Registry for Alzheimer's Disease (CERAD). Part I. Clinical and neuropsychological assessment of Alzheimer's disease. Neurology 1989;39:1159-65.

10. Wechsler D. Wechsler adult intelligence scales:In. The Psychological Corporation. 3rd ed, 1997.

11. Wechsler D. Wechsler Memory Scale-Fourth Edition (WMS-IV) technical and interpretive manual: Pearson, 2009.

12. Rosen WG, Mohs RC, Davis KL. A new rating scale for Alzheimer's disease. Am J Psychiatry 1984;141:1356-64.

13. Ruesch J. Intellectual impairment in head injuries. American Journal of Psychiatry 1944;100:480-96.

14. Hall $\mathrm{K}$, Hendrie $\mathrm{H}$, Brittain $\mathrm{H}$, et al. The development of a dementia screening interview in two distinct languages. Int $J$ Methods Psychiatr Res 1993;3:1-28.

15. Raven J. The Raven's progressive matrices: change and stability over culture and time. Cogn Psychol 2000;41:1-48.

16. Agrigoroaei S, Lachman ME. Cognitive functioning in midlife and old age: combined effects of psychosocial and behavioral factors. $J$ Gerontol B Psychol Sci Soc Sci 2011;66 Suppl 1(Suppl 1):i130-i140.

17. Fisher G, McArdle J, McCammon R, et al. New measures of fluid intelligence in the HRS. Institute for Social Research, University of Michigan 2013 https://hrs.isr.umich.edu/sites/default/files/biblio/dr027b.pdf.

18. Mesulam M. Principles of behavioral neurology: FA Davis Company, 1985.

19. Tripathi R, Kumar JK, Bharath S, et al. Clinical validity of NIMHANS neuropsychological battery for elderly: A preliminary report. Indian $J$ Psychiatry 2013;55:279-82

20. Jorm AF, Jacomb PA. The Informant Questionnaire on Cognitive Decline in the Elderly (IQCODE): socio-demographic correlates, reliability, validity and some norms. Psychol Med 1989;19:1015-22.

21. Blessed G, Tomlinson BE, Roth M. The association between quantitative measures of dementia and of senile change in the cerebral grey matter of elderly subjects. $\mathrm{Br} J$ Psychiatry 1968;114:797-811.

22. Prince $M$, Ferri CP, Acosta D, et al. The protocols for the $10 / 66$ dementia research group population-based research programme. BMC Public Health 2007;7:165.

23. Beck AT, Epstein N, Brown G, et al. An inventory for measuring clinical anxiety: psychometric properties. J Consult Clin Psychol 1988;56:893-7.

24. Radloff L. The CES-D scale: a self-report depression scale for research in the general population. Appl Psychol Meas 1977:1:385-401.

25. Podsiadlo D, Richardson S. The timed "Up \& Go": a test of basic functional mobility for frail elderly persons. J Am Geriatr Soc 1991:39:142-8

26. ATS Committee on Proficiency Standards for Clinical Pulmonary Function Laboratories. ATS statement: guidelines for the six-minute walk test. Am J Respir Crit Care Med 2002;166:111-7.

27. Vellas B, Guigoz Y, Garry PJ, et al. The Mini Nutritional Assessment (MNA) and its use in grading the nutritional state of elderly patients. Nutrition 1999;15:116-22.

28. Weir DR, Wallace RB, Langa KM, et al. Reducing case ascertainment costs in U.S. population studies of Alzheimer's disease, dementia, and cognitive impairment-Part 1. Alzheimers Dement 2011;7:94-109.

29. Becker JT, Duara R, Lee CW, et al. Cross-validation of brain structural biomarkers and cognitive aging in a community-based study. Int Psychogeriatr 2012;24:1065-75.

30. Morris JC. The Clinical Dementia Rating (CDR): current version and scoring rules. Neurology 1993;43:2412-4.

31. Fleiss J, Levin B, Paik M. Statistical methods for rates and proportions. 3rd ed: John Wiley \& Sons, Inc, 2003

32. Hurd MD, Martorell P, Delavande A, et al. Monetary costs of dementia in the United States. N Engl J Med 2013;368:1326-34.

33. Ofstedal M, Fisher G, Herzog A. Documentation of cognitive functioning measures in the health and retirement study (DR-006). Institute for Social Research, University of Michigan 2005 http:// hrsonline.isr.umich.edu/sitedocs/userg/dr-006.pdf.

34. Folstein MF, Folstein SE, McHugh PR. "Mini-mental state". A practical method for grading the cognitive state of patients for the clinician. J Psychiatr Res 1975;12:189-98.

35. Nasreddine ZS, Phillips NA, Bédirian V, et al. The montreal cognitive assessment, MoCA: a brief screening tool for mild cognitive impairment. J Am Geriatr Soc 2005;53:695-9. 
36. Mattis S. Dementia rating scale professional manual. Psychological Assessment Resources 1988.
37. De Renzi E, VIGNOLO LA. The token test: A sensitive test to detect receptive disturbances in aphasics. Brain 1962;85:665-78. 